\title{
Fuchs, Christian: Kommunikation und Kapitalismus: Eine kritische Theorie
}

Stuttgart: UTB 2020. 501 Seiten. Preis: $€ 25,00$

\author{
Wolfgang Seufert
}

Angenommen: 3. Juni 2021 / Online publiziert: 24. Juni 2021

(C) Der/die Autor(en) 2021

Mit dem in der utb-Lehrbuchreihe erschienen Band will Christian Fuchs laut Klappentext eine Einführung in die kritische Theorie der Kommunikation und in die Kritik der politischen Ökonomie der Kommunikation geben. Er will vor allem folgende fünf Fragen beantworten: „Was ist Kommunikation? Was sind die Rollen der Kommunikation in der Gesellschaft? Wie kann die Kommunikation mit Hilfe eines materialistischen Ansatzes (kommunikativer Materialismus) kritisch analysiert werden? Was sind die Rollen der Kommunikation im Kapitalismus? Welche Alternative zur kapitalistischen Kommunikation gibt es?“ Die Art der Fragen macht deutlich, dass Fuchs „Kommunikation“ primär aus einer Makro-Perspektive analysiert. Es geht ihm um die Gesamtheit aller Kommunikationsprozesse und deren Einbettung in die Gesellschaft als Ganzes bzw. um spezifische Wirkungsweisen der Kommunikation in den drei gesellschaftlichen Teilsystemen „Wirtschaft“, „Politik“ und „Kultur“. Sein Ausgangspunkt ist dabei eine Gesellschaftstheorie, die er im Einleitungskapitel als aristotelischen, dialektischen und humanistischen Marxismus charakterisiert. Der Band hat insgesamt 15 Kapitel und greift eine Fülle unterschiedlichster Themen auf. Im Einleitungskapitel werden Ziele, die der Autor mit dem Buch verfolgt sowie die eigenen Positionen stark verdichtet dargestellt. Der anschließende Haupttext ist in drei Abschnitte gegliedert:

In Teil I („Grundlagen des kommunikativen Materialismus“) entwickelt Fuchs (1) seine philosophischen Grundlagen, im Wesentlichen eine Bezugnahme auf den Materiebegriff von Aristoteles und Hegels dialektischer Entwicklungslogik, bei der Antagonismen auf einer höheren Stufe aufgehoben werden; (2) eine auf Marx basierende Gesellschaftstheorie, in der das Organisationssystem der Wirtschaft (aktuell:

Prof. Dr. Wolfgang Seufert $(\bowtie)$

Institut für Kommunikationswissenschaft, Friedrich-Schiller-Universität Jena,

Ernst-Abbe-Platz 8, 07743 Jena, Deutschland

E-Mail: w.seufert@uni-jena.de 
das Prinzip der Ausbeutung von Lohnarbeit durch die Eigentümer der Produktionsmittel) auch Herrschaftsverhältnisse in Politik und Kultur prägt; (3) das Verständnis von Kommunikation als wesentlichen Aspekt menschlicher Werktätigkeit, zu der u.a. die ,kommunikative Koordination des Produktionsprozesses, der Einsatz von Kommunikationstechnologien in Produktion, Distribution und dem Konsum“ gehören. „Kooperative Kommunikation“ (re-)produziert nach Fuchs aber auch soziale Beziehungen, soziale Strukturen, soziale Systeme usw. Aus individuellen Erfahrungen, Ideen und Erkenntnissen werden geteilte Bedeutungen und gesellschaftliche Wissensbestände, einschließlich kollektiver politischer Weltanschauungen (als Teil der Politik) und kollektiver Identitäten (als Teil der Kultur).

Teil II (,Kommunikation in der Kapitalistischen Gesellschaft“) vertieft Aspekte der Kommunikation im Wirtschaftssystem und im politischen System. Schwerpunkte sind u.a. (1) eine Abgrenzung der ,kapitalistischen Kommunikationsindustrie“ - neben den Inhaltsproduzenten auch die gesamte IT-Wirtschaft - anhand verschiedener „Akkumulationsmodelle“; (2) eine (letztlich ablehnende) Auseinandersetzung mit der Idee revolutionärer Medientechnologien als treibende Kraft der Gesellschaftsentwicklung; (3) die Diskussion der Tragfähigkeit des Konzeptes der Informationsgesellschaft zur Beschreibung des zunehmenden Anteils an Informations- und Kreativarbeit; (4) die Produktion von Ideologie (,falsches Bewusstseins“) im Interesse der Herrschenden aufgrund deren Kontrolle weiter Teile der Kulturindustrie; (5) das antagonistische Verhältnis von Globalisierung (als Versuch einer Krisenbewältigung durch Lohnkostensenkung bei Universalisierung der kapitalistischen Ausbeutungslogik) und den Ideologien des Nationalismus bzw. Rassismus. Letztere versuchen, mit der Fiktion biologischer oder kultureller Gemeinschaften den Widerspruch zwischen Kapital und Arbeit zu überdecken.

Teil III („Die materialistische Transformation des kommunikativen Kapitalismus") ist der politischen Praxis gewidmet. Fuchs beschreibt als erstrebenswerte sozialistische Alternative ein Organisationsprinzip der Wirtschaft, in dem die Produktion grundlegender „Güter, die alle Menschen brauchen, um ein gutes Leben zu führen“ unter kollektiver Kontrolle („Gemeingüter“) steht. In einem kommunikationspolitischen 10-Punkte-Programm werden u.a. die Förderung öffentlichrechtlicher Medien bzw. in Kooperativen organisierte Alternativmedien gefordert, da sie keiner kapitalistischen Verwertungslogik unterliegen und damit das Potenzial zur Schaffung „kritischen Bewusstseins“ haben, aber auch strikte Datenschutzregeln, die eine Dauerbeobachtung des Konsum- bzw. Arbeitsverhaltens verhindern sollen. Das Schlusskapitel ist vor allem eine Auseinandersetzung mit Habermas' „Theorie kommunikativen Handelns“, die nach Fuchs als „dualistischer Ansatz“ (verständigungsorientierte/erfolgsorientierte Kommunikation usw.) die Illusion eines kommunikativen Interessenausgleichs zwischen Kapitalisten und Lohnarbeitern erzeuge.

Die Vorgehensweise des Autors, die eigene Position als Gegenentwurf zu ,,antihumanistischen“ Makro-Theorien ohne menschliche Akteure (Luhmanns Systemtheorie, Foucaults Diskurstheorie, Labours Akteurs-Netzwerktheorie, McLuhans Theorie medientechnikdeterminierter Entwicklungsepochen usw.), aber auch als Gegenposition zu anderen „,kritischen“ Theorien zu entwickeln - wobei dabei oft eine marxistische Insiderdiskussion geführt wird - ist für ein „Lehrbuch“ bzw. eine „Ein- 
führung“ äußerst anspruchsvoll. Zwar gibt es am Ende jedes Kapitels Zusammenfassungen der wichtigsten Argumente, ein tieferes Verständnis des Textes setzt aber mindestens Kenntnisse über das Spektrum gesellschaftlicher Makro-Theorien voraus. Verständnisprobleme dürfte auch die dialektische Methodik bereiten, auf die Fuchs immer wieder verweist, zumal die erläuternden Abbildungen häufig eher „Wechselwirkungen“ als Antagonismen zeigen. Auch die marxistische Terminologie, beispielsweise die extensiv verwendeten Begriffe ,gesellschaftliche Totalität“" oder „Verdinglichung“/,Entfremdung“ sind gewöhnungsbedürftig. Insbesondere im Teil zur Transzendenz des Kapitalismus werden Argumente zudem mit sozialistischer Klassenkampfrhetorik vermischt.

Welchen Nutzen können nun Wirtschaftswissenschaftler, Politikwissenschaftler oder Kommunikationswissenschaftler, die sich mit der Analyse der Wirkungen bzw. der Veränderung von Strukturen im Medienbereich empirisch oder auf anderer Theoriegrundlage beschäftigten, aus der Lektüre ziehen? Da Fuchs explizit auf eine Diskussion ,bürgerlicher“ Theorien verzichtet, gibt es wenig Anknüpfungspunkte für eine fruchtbare Debatte. Empirie findet sich zwar in Form einfacher deskriptiver Statistik als Datensammlung zum Beleg hoher Medienkonzentration, eines wachsenden Anteils von Informations- und Kreativarbeit, oder von Globalisierungstendenzen. Eine empirische Falsifizierbarkeit des eigenen Ansatzes ist allerdings nicht beabsichtigt. So kann am Ende, ökonomisch gesprochen, nur per „Beauty Test“ entschieden werden, ob der von Fuchs vertretene gesellschaftstheoretische Ansatz überzeugender oder ,richtiger“ als andere Meta-Theorien ist. Inwieweit diese Überzeugungsarbeit mit dem Buch gelingt, bleibt fraglich.

Funding Open Access funding enabled and organized by Projekt DEAL.

Open Access Dieser Artikel wird unter der Creative Commons Namensnennung 4.0 International Lizenz veröffentlicht, welche die Nutzung, Vervielfältigung, Bearbeitung, Verbreitung und Wiedergabe in jeglichem Medium und Format erlaubt, sofern Sie den/die ursprünglichen Autor(en) und die Quelle ordnungsgemäß nennen, einen Link zur Creative Commons Lizenz beifügen und angeben, ob Änderungen vorgenommen wurden.

Die in diesem Artikel enthaltenen Bilder und sonstiges Drittmaterial unterliegen ebenfalls der genannten Creative Commons Lizenz, sofern sich aus der Abbildungslegende nichts anderes ergibt. Sofern das betreffende Material nicht unter der genannten Creative Commons Lizenz steht und die betreffende Handlung nicht nach gesetzlichen Vorschriften erlaubt ist, ist für die oben aufgeführten Weiterverwendungen des Materials die Einwilligung des jeweiligen Rechteinhabers einzuholen.

Weitere Details zur Lizenz entnehmen Sie bitte der Lizenzinformation auf http://creativecommons.org/ licenses/by/4.0/deed.de.

Prof. Dr. Wolfgang Seufert ist Professor für Kommunikationswissenschaft mit dem Schwerpunkt Ökonomie und Organisation der Medien an der Friedrich-Schiller-Universität Jena. 